\title{
Evidence for acoustic-like plasmons on epitaxial graphene on $\operatorname{Pt}(111)$
}

\author{
A. Politano, ${ }^{1}$ A. R. Marino, ${ }^{2}$ V. Formoso,${ }^{2,3}$ D. Farías,${ }^{1,4}$ R. Miranda,${ }^{1,4,5}$ and G. Chiarello ${ }^{2,6, *}$ \\ ${ }^{1}$ Departamento de Física, Universidad Autónoma de Madrid, Campus Universitario de Cantoblanco, 28049 Madrid, Spain \\ ${ }^{2}$ Dipartimento di Fisica, Università degli Studi della Calabria, 87036 Rende (Cs), Italy \\ ${ }^{3}$ Laboratorio Regionale Licryl, Liquid Crystal Laboratory, 87036 Rende (Cs), Italy \\ ${ }^{4}$ Instituto de Ciencia de Materiales "Nicolás Cabrera", Universidad Autónoma de Madrid, Cantoblanco, 28049 Madrid, Spain \\ ${ }^{5}$ Instituto Madrileño de Estudios Avanzados (IMDEA) en Nanociencia, Cantoblanco, 28049 Madrid, Spain \\ ${ }^{6}$ Consorzio Nazionale Interuniversitario di Scienze Fisiche della Materia, via della Vasca Navale, 84, 00146 Roma, Italy
}

(Received 24 February 2011; revised manuscript received 9 May 2011; published 1 July 2011)

\begin{abstract}
The dispersion and the damping of the sheet plasmon in a graphene monolayer grown on $\operatorname{Pt}(111)$ have been studied by using angle-resolved electron energy loss spectroscopy. We found that the dispersion relation of the plasmon mode confined in the graphene sheet is linear, as a consequence of the screening by the metal substrate. Present results demonstrate that the presence of an underlying metal substrate could have striking consequences on the plasmon propagation even in the case of a system which exhibits a weak graphene-substrate interaction. Moreover, we found that Landau damping essentially occurs via interband excitations starting above the Fermi wave vector. On the contrary, intraband transitions do not have a significant influence on the collective mode.
\end{abstract}

DOI: 10.1103/PhysRevB.84.033401

PACS number(s): 79.20.Uv, 73.20.Mf, 68.65.Pq

Low-energy collective excitations in graphene are attracting much interest in recent years ${ }^{1-8}$ as they influence many of the peculiar properties of graphene samples. In particular, the dispersion and damping of plasmons in epitaxial graphene have recently been studied for the case of graphene deposited on $\operatorname{SiC}(0001)$ (Refs. 2 and 4) and $\operatorname{Ir}(111){ }^{6}$ The understanding of plasmonic excitations of graphene plays a key role in tailoring the properties of novel graphene-based devices. ${ }^{9}$ Among graphene systems, the epitaxial growth of monolayer graphene (MLG) on $\operatorname{Pt}(111)$ is particularly interesting ${ }^{10-14}$ as a consequence of the weak graphene-Pt interaction, ${ }^{11}$ in contrast with MLG grown on other transition-metal substrates. ${ }^{15,16}$ In fact, the graphene-Pt distance (3.30 $\AA$ ) lies close to the $c$-axis spacing in graphite. The electronic structure of MLG on $\mathrm{Pt}(111)$ resembles that of isolated graphene. ${ }^{10}$ In particular, the linear dispersion of $\pi$ bands in the so-called Dirac cones, which gives rise to many manifestations of massless Dirac fermions, is preserved. Angle-resolved photoemission spectroscopy (ARPES) experiments ${ }^{10}$ do not show any remarkable hybridization of graphene $\pi$ states with metal $d$ states. They just represent a superposition of graphene and metal-derived states, with minimal interaction between them. The MLG on $\mathrm{Pt}(111)$ is hole doped by charge transfer to the Pt substrate. ${ }^{12}$ The Fermi energy $E_{F}$ of the graphene layer shifts $0.30 \pm$ $0.15 \mathrm{eV}$ below the Dirac-energy crossing point of the bands, with the Fermi wave vector $k_{F}=0.09 \AA^{-1}$.

Epitaxial graphene on $\operatorname{Pt}(111)$ thus behaves as an ideal 2D system, sustaining a purely 2D electron gas (2DEG) system whose collective excitations (plasmon modes) are able to propagate along the sheet. The dielectric response of the 2DEG system is determined by plasmon dispersion, which could be measured by high-resolution electron energy loss spectroscopy (HREELS).

The 2D plasmon, characterized by its square-root-like dispersion, has been predicted ${ }^{17}$ and observed in metal layers on semiconductors. ${ }^{18}$ On the other hand, the acoustic surface plasmon (ASP) with a linear dispersion was demonstrated to exist on semiconductor quantum wells with two interacting quantum well minibands. ${ }^{19}$ Successively, ASP has been exper- imentally revealed on $\mathrm{Be}(0001)$ (Ref. 20) and on noble-metal surfaces. ${ }^{21,22}$ The acoustic-like dispersion is a consequence of the combination of the nonlocality of the 3D response and the spillout of the 3D electron density into the vacuum, both providing incomplete screening of the $2 \mathrm{D}$ electron-density oscillations. $^{23}$

Previous measurements on $\mathrm{MLG} / \mathrm{SiC}(0001)$ showed a nonlinear dispersion for the sheet plasmon in MLG. Such behavior could be described by the Stern's model. ${ }^{24}$ It is interesting to study the behavior of collective excitations of MLG grown on a metal substrate in order to shed light on the screening mechanisms of the sheet plasmon in the presence of an underlying metal substrate. Present measurements by HREELS show a linear dispersion for the sheet plasmon in MLG/Pt(111). Our results indicate that the sheet plasmon of MLG survives up to a high energy, i.e., $3 \mathrm{eV}$. This is a consequence of the fact that intraband excitations have negligible influence on the propagation of the plasmon mode. On the other hand, the dispersion curve of the sheet plasmon overlaps with the continuum of interband transitions above the Fermi wave vector. This broadens the plasmon peak but does not cause its disappearance (in agreement with predictions in Ref. 5), in contrast with the behavior found for ordinary sheet plasmons in 2DEG and ASP.

Experiments were carried out in an ultra-high vacuum (UHV) chamber operating at a base pressure of $5 \times 10^{-11} \mathrm{mbar}$. The sample was a single crystal of $\operatorname{Pt}(111)$. The substrate was cleaned by repeated cycles of ion sputtering and annealing at $1300 \mathrm{~K}$. Surface cleanliness and order were checked using Auger electron spectroscopy (AES) and low-energy electron diffraction (LEED) measurements, respectively. Graphene was obtained by dosing ethylene onto the clean $\operatorname{Pt}(111)$ substrate kept at $1150 \mathrm{~K}$. The completion of the first layer was reached upon an exposure of $3 \times 10^{-8}$ mbar for $10 \mathrm{~min}(24 \mathrm{~L}, 1 \mathrm{~L}=$ $1.33 \times 10^{-6}$ mbar s). After removing the $\mathrm{C}_{2} \mathrm{H}_{4}$ gas from the chamber, the temperature was held at $1150 \mathrm{~K}$ for further $60 \mathrm{~s}$. In these conditions, the observed LEED pattern is similar to the one shown in Ref. 13 for MLG/Pt(111). Furthermore, the characterization of the MLG has been carried out by 
identifying phonon modes which are fingerprint of graphene formation. ${ }^{25}$ HREEL experiments were performed by using an electron energy loss spectrometer (Delta 0.5, SPECS). The energy resolution of the spectrometer was degraded to $5 \mathrm{meV}$ so as to increase the signal-to-noise ratio of loss peaks. Dispersion of the loss peaks, i.e., $E_{\text {loss }}\left(q_{\|}\right)$, was measured by moving the analyzer while keeping the sample and the monochromator in a fixed position. To measure plasmon dispersion, values for the parameters $E_{p}$, impinging energy, and $\theta_{i}$, the incident angle, were chosen so as to obtain the highest signal-to-noise ratio. The primary beam energy used for the dispersion, $E_{p}=7-12 \mathrm{eV}$, provided, in fact, the best compromise among surface sensitivity, the highest cross section for mode excitation and $q_{\|}$resolution. As

$$
\hbar \overrightarrow{q_{\|}}=\hbar\left(\overrightarrow{k_{i}} \sin \theta_{i}-\overrightarrow{k_{s}} \sin \theta_{s}\right)
$$

the parallel momentum transfer $q_{\|}$depends on $E_{p}, E_{\text {loss }}, \theta_{i}$, and $\theta_{s}$ according to

$$
q_{\|}=\frac{\sqrt{2 m E_{p}}}{\hbar}\left(\sin \theta_{i}-\sqrt{1-\frac{E_{\text {loss }}}{E_{p}}} \sin \theta_{s}\right),
$$

where $E_{\text {loss }}$ is the energy loss and $\theta_{s}$ is the electron scattering angle. ${ }^{26}$

Accordingly, the integration window in reciprocal space $^{27}$ is

$$
\Delta q_{\|} \approx \frac{\sqrt{2 m E_{p}}}{\hbar}\left(\cos \theta_{i}+\sqrt{1-\frac{E_{\mathrm{loss}}}{E_{p}}} \cos \theta_{s}\right) \alpha,
$$

where $\alpha$ is the angular acceptance of the apparatus, ${ }^{27}\left( \pm 0.5^{\circ}\right.$ in our case). To obtain the energies of loss peaks, a polynomial background was subtracted from each spectrum. The resulting spectra were fitted by a Gaussian line shape (not shown herein). All measurements were made at room temperature.

Measurements were performed for both symmetry directions $(\bar{\Gamma}-\bar{K}$ and $\bar{\Gamma}-\bar{M})$, but no remarkable differences were recorded as a consequence of the existence of differently oriented domains on the sample, as observed in previous low-energy electron microscopy experiments. ${ }^{10}$ Loss measurements of $\mathrm{MLG} / \mathrm{Pt}(111)$ recorded as a function of the scattering angle $\theta_{s}$ are reported in Fig. 1. HREEL spectra show a low-energy feature which develops and disperses up to $3 \mathrm{eV}$ as a function of the scattering angle. This resonance exhibits a clear linear dispersion and its frequency approaches zero in the long-wavelength limit. We assign it to the sheet plasmon of MLG, in agreement with theoretical ${ }^{1,3,5,28,29}$ and experimental ${ }^{2,4,24}$ results.

The dispersion of the sheet plasmon for MLG on $\mathrm{SiC}(0001)$ well agrees with Stern's ${ }^{17}$ prediction $\left(\omega \propto \sqrt{q_{\|}}\right)$. However, the plasmon dispersion recorded in our experiments (Fig. 2) is well described by a linear relationship, as in the case of ASP on bare metal surfaces: ${ }^{20-22}$

$$
\hbar \omega_{2 D}=A q_{\|},
$$

where $A=(7.4 \pm 0.1) \mathrm{eV} \cdot \AA$

The sheet plasmon with a linear dispersion owes its existence to the interplay of the underlying metal substrate with the $\pi$-charge density in the MLG in the same region of space. It resembles the ASP in metal surfaces that support a partially

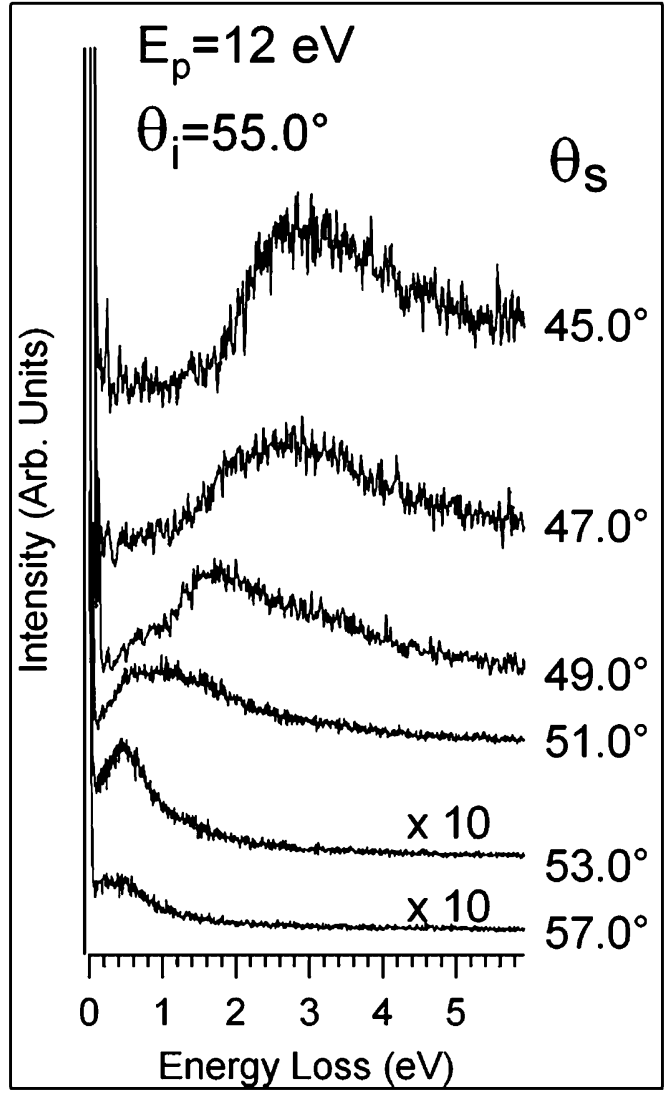

FIG. 1. HREEL spectra of MLG/Pt(111) acquired as a function of the scattering angle. The incident angle is $55.0^{\circ}$. The impinging energy $E_{p}$ is $12 \mathrm{eV}$.

occupied surface state band within a wide bulk energy gap..$^{30,31}$ The nonlocal character of the dielectric function ${ }^{28}$ and the screening processes in graphene $\mathrm{e}^{32,33}$ prevent the sheet plasmon from being screened out by the 3D bulk states of $\operatorname{Pt}(111)$. Recently, Horing ${ }^{28}$ predicted that the linear plasmon in graphene systems may arise from the Coulombian interaction between the native sheet plasmon $\left(\omega \propto \sqrt{q_{\|}}\right)$in MLG and the surface plasmon of a nearby thick substrate hosting a semi-infinite plasma. Calculations taking into account the electronic response of the Pt substrate could in principle put this effect in evidence, but this is not trivial due to the existence of a Moire reconstruction in the MLG lattice on top of the $\mathrm{Pt}(111)$ substrate. The slope of the dispersion relation of the sheet plasmon in MLG/Pt(111) and the ones of acoustic-like excitations provide information about group velocities of the plasmon mode. We found that the group velocity of the sheet plasmon in MLG/Pt(111) $\left(1.1 \pm 0.2 \times 10^{6} \mathrm{~m} / \mathrm{s}\right)$ is similar to the ones calculated for ASP. ${ }^{34}$ The group velocity of the sheet plasmon in MLG/Pt(111) is about 2 orders of magnitude lower than the speed of light, thus its direct excitation by light is not possible. However, nanometer-size objects at surfaces, such as atomic steps or molecular structures, can allow coupling between sheet plasmon and light. The linear behavior of its dispersion implies that both phase and group velocities of the collective excitation are the same, so signals can be transmitted undistorted along the surface. Hence, this finding could be of significant importance in future graphene-based nano-optical 


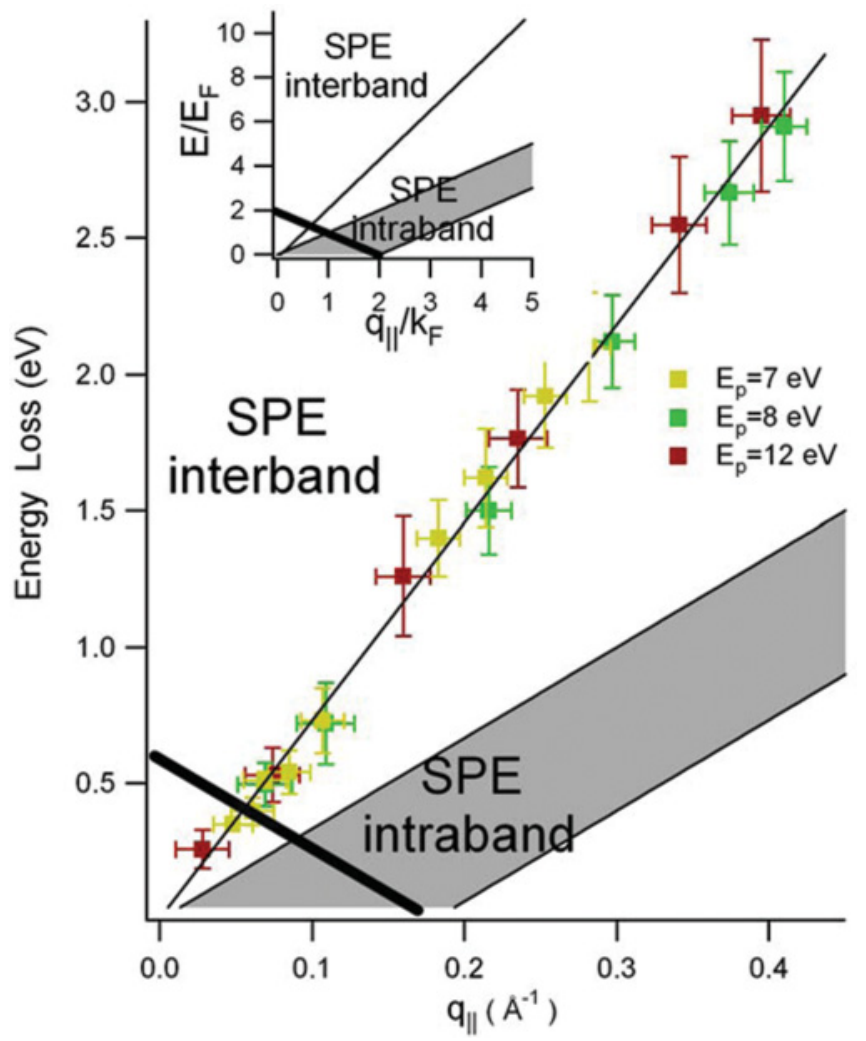

FIG. 2. (Color online) Plasmon dispersion in MLG/Pt(111). Data have been acquired for three different impinging energies. The thin solid line represents the best fit for experimental points. The dashed area indicates the continuum of intraband SPEs. The thick solid line represents the boundary for the continuum of interband SPEs. The plasmon mode enters the Landau damping regime by interband electron-hole excitations when its dispersion relation intercepts the boundary for the continuum of interband SPEs. In the inset, the curves are reported with respect to the dimensionless units $E / E_{F}$ and $q_{\|} / k_{F}$.

devices, especially if we have in mind that the Moiré pattern of MLG on metal substrates offers a naturally nanostructured system. ${ }^{15,35}$ In Fig. 2, we also show the electron-hole continuum or single-particle excitation (SPE) region, which determines the absorption (Landau damping) of the external field at given frequency. It was calculated on the basis of results in Refs. 5, 36, and 37 by substituting the values of $E_{F}$ and $k_{F}$ for MLG/Pt(111) obtained by ARPES. ${ }^{10}$ For a normal 2D system, only indirect transition is possible within the band. However, for graphene, both intraband and interband transitions are possible, and the boundaries are given in Fig. 2. Due to the phase-space restriction, the interband SPE continuum has a gap at small momenta. For $q_{\|}=0$, the transition is not allowed at $0<E<2 E_{F}$. If the collective mode enters the SPE continuum, the plasmon mode can be damped. The plasmon lies inside the interband SPE continuum, thus decaying into electron-hole pairs, above the Fermi wave vector. Plasmon can propagate without damping only in the region (see Fig. 2) not included in the continuum of SPE (interband and intraband). Such considerations are fully confirmed by the analysis of the full-width at half maximum (FWHM) of the plasmon peak

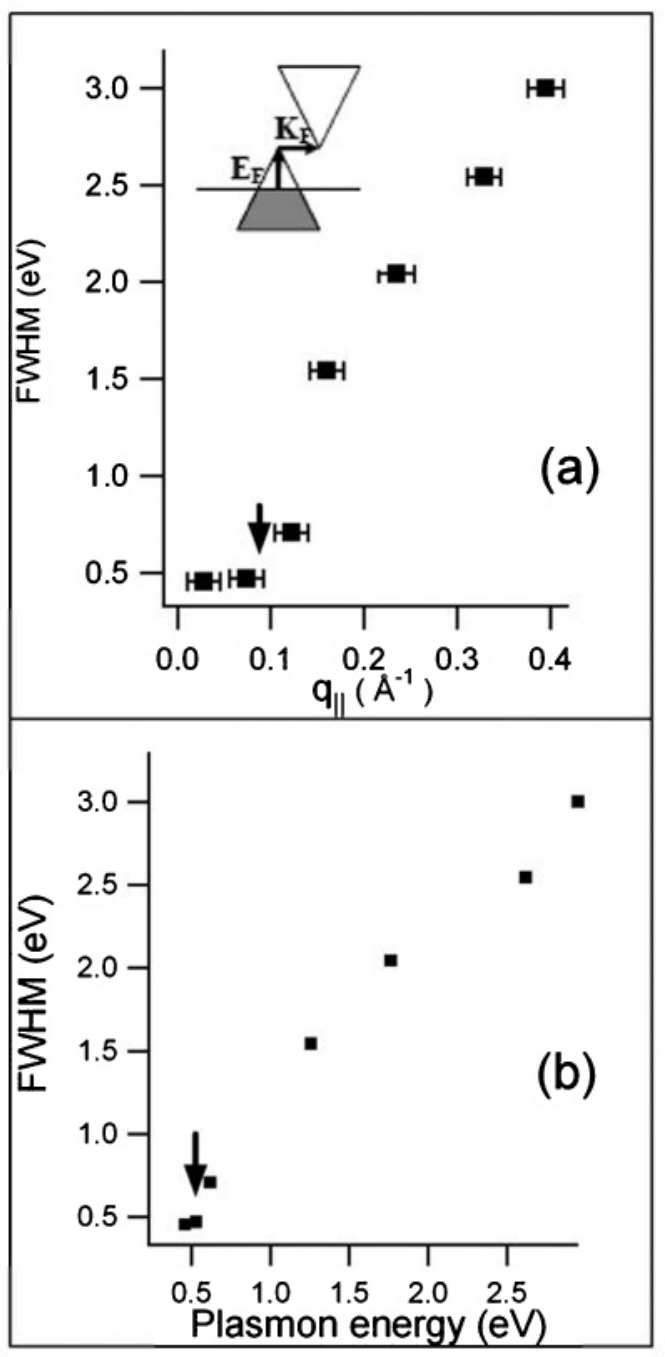

FIG. 3. FWHM of the plasmon peak as a function of (a) the parallel momentum transfer $q_{\|}$and of (b) the plasmon energy. The inset in the top panel shows the origin of interband SPEs from $\pi$ to $\pi^{*}$ bands. The Fermi wave vector represents the onset where plasmon enters the damping region.

as a function of both $q_{\|}$[Fig. 3(a)] and the plasmon energy [Fig. 3(b)]. Landau damping for the MLG sheet plasmon occurs for momenta above the Fermi wave vector (about $0.09 \AA^{-1}$ ) and for energies above $0.5 \mathrm{eV}$, as revealed by the sudden increase of the FWHM. Interestingly, the sheet plasmon does not enter into the intraband SPE continuum and it exists for all wave vectors. By contrast, for ASP, Landau damping occurs via intraband transitions and the plasmon mode exists only up to a few hundreds $m e{ }^{20-22}$ On the other hand, for MLG on $\mathrm{SiC}(0001)$, the FWHM continuously increases with the momentum. ${ }^{2,24}$ For such system, it has been shown ${ }^{2}$ that the existence of steps or grain boundaries is a source of strong damping, while the dispersion is rather insensitive to defects.

In conclusion, we found a linear dispersion of the sheet plasmon in MLG on $\mathrm{Pt}(111)$. Such behavior is attributed to the nonlocal screening of the plasmon mode of MLG caused by the underlying Pt substrate. Due to its low energy and its 
linear dispersion, the sheet plasmon is expected to play an important role in graphene dynamics. This could be especially relevant for future graphene-based nano-optical devices, since the rippled, nanostructured surface of MLG on metal substrates (see Refs. 15 and 35) provides an interesting scenario to couple acoustic surface plasmons and light. We have also shown that Landau damping occurs via interband transitions starting above the Fermi wave vector.

A.P. thanks the Ministerio de Educación y Ciencia (MEC) for salary support. We are grateful to Dr. Marco Polini for his suggestions and for a critical review of the manuscript. *gennaro.chiarello@fis.unical.it

${ }^{1}$ N. J. M. Horing, IEEE Trans. Nanotechnol. 9, 679 (2010).

${ }^{2}$ T. Langer, J. Baringhaus, H. Pfnür, H. W. Schumacher, and C. Tegenkamp, New J. Phys. 12, 033017 (2010).

${ }^{3}$ N. J. M. Horing, Phys. Rev. B 80, 193401 (2009).

${ }^{4}$ C. Tegenkamp, H. Pfnür, T. Langer, J. Baringhaus, and H. W. Schumacher, J. Phys. Condens. Matter 23, 012001 (2011).

${ }^{5}$ M. Polini, R. Asgari, G. Borghi, Y. Barlas, T. Pereg-Barnea, and A. H. MacDonald, Phys. Rev. B 77, 081411 (2008).

${ }^{6}$ H. Pfnür, T. Langer, J. Baringhaus, and C. Tegenkamp, J. Phys. Condens. Matter 23, 112204 (2011).

${ }^{7}$ Yu Liu and R. F. Willis, Phys. Rev. B 81, 081406(R) (2010).

${ }^{8}$ T. Langer, H. Pfnür, H. W. Schumacher, and C. Tegenkamp, Appl. Phys. Lett. 94, 112106 (2009).

${ }^{9}$ A. Bostwick, F. Speck, T. Seyller, K. Horn, M. Polini, R. Asgari, A. H. MacDonald, and E. Rotenberg, Science 328, 999 (2010).

${ }^{10}$ P. Sutter, J. T. Sadowski, and E. Sutter, Phys. Rev. B 80, 245411 (2009).

${ }^{11}$ A. B. Preobrajenski, M. L. Ng, A. S. Vinogradov, and N. Martensson, Phys. Rev. B 78, 073401 (2008).

${ }^{12}$ M. Gao, Y. Pan, C. Zhang, H. Hu, R. Yang, H. Lu, J. Cai, S. Du, F. Liu, and H. J. Gao, Appl. Phys. Lett. 96, 053109 (2010).

${ }^{13}$ M. Gao, Y. Pan, L. Huang, H. Hu, L. Z. Zhang, H. M. Guo, S. X. Du, and H. J. Gao, Appl. Phys. Lett. 98, 033101 (2011).

${ }^{14}$ G. Otero, C. Gonzalez, A. L. Pinardi, P. Merino, S. Gardonio, S. Lizzit, M. Blanco-Rey, K. Van de Ruit, C. F. J. Flipse, J. Méndez, P. L. de Andrés, and J. A. Martin-Gago, Phys. Rev. Lett. 105, 216102 (2010).

${ }^{15}$ B. Borca, S. Barja, M. Garnica, M. Minniti, A. Politano, J. M. Rodriguez-García, J. J. Hinarejos, D. Farías, A. L. Vázquez de Parga, and R. Miranda, New J. Phys. 12, 093018 (2010).

${ }^{16}$ D. Farías, A. M. Shikin, K. H. Rieder, and Y. S. Dedkov, J. Phys. Condens. Matter 11, 8453 (1999).

${ }^{17}$ F. Stern, Phys. Rev. Lett. 18, 546 (1967).

${ }^{18}$ T. Nagao, T. Hildebrandt, M. Henzler, and S. Hasegawa, Phys. Rev. Lett. 86, 5747 (2001).
${ }^{19}$ Y. Chen, J. C. Hermanson, and G. J. Lapeyre, Phys. Rev. B 39, 12682 (1989).

${ }^{20}$ B. Diaconescu, K. Pohl, L. Vattuone, L. Savio, P. Hofmann, V. M. Silkin, J. M. Pitarke, E. V. Chulkov, P. M. Echenique, D. Farías, and M. Rocca, Nature 448, 57 (2007).

${ }^{21}$ K. Pohl, B. Diaconescu, G. Vercelli, L. Vattuone, V. M. Silkin, E. V. Chulkov, P. M. Echenique, and M. Rocca, Europhys. Lett. 90, 57006 (2010).

${ }^{22}$ S. J. Park and R. E. Palmer, Phys. Rev. Lett. 105, 016801 (2010).

${ }^{23}$ J. M. Pitarke, V. U. Nazarov, V. M. Silkin, E. V. Chulkov, E. Zaremba, and P. M. Echenique, Phys. Rev. B 70, 205403 (2004).

${ }^{24}$ Y. Liu, R. F. Willis, K. V. Emtsev, and T. Seyller, Phys. Rev. B 78, 201403 (2008).

${ }^{25}$ D. Farías, K. H. Rieder, A. M. Shikin, V. K. Adamchuk, T. Tanaka, and C. Oshima, Surf. Sci. 454, 437 (2000).

${ }^{26}$ M. Rocca, Surf. Sci. Rep. 22, 1 (1995).

${ }^{27}$ A. Politano, R. G. Agostino, E. Colavita, V. Formoso, and G. Chiarello, J. Nanosci. Nanotechnol. 9, 3932 (2009).

${ }^{28}$ N. J. M. Horing, Philos. Trans. R. Soc. London A 368, 5525 (2010).

${ }^{29}$ Y. Gao and Z. Yuan, Solid State Commun. 151, 1009 (2011).

${ }^{30}$ V. M. Silkin, A. Garcia-Lekue, J. M. Pitarke, E. V. Chulkov, E. Zaremba, and P. M. Echenique, Europhys. Lett. 66, 260 (2004).

${ }^{31}$ V. M. Silkin, J. M. Pitarke, E. V. Chulkov, and P. M. Echenique, Phys. Rev. B 72, 115435 (2005).

${ }^{32}$ M. van Schilfgaarde and M. I. Katsnelson, Phys. Rev. B 83, 081409 (2011).

${ }^{33}$ J. Yan, K. S. Thygesen, and K. W. Jacobsen, Phys. Rev. Lett. 106, 146803 (2011)

${ }^{34}$ V. M. Silkin, A. Garcia-Lekue, J. M. Pitarke, E. V. Chulkov, E. Zaremba, and P. M. Echenique, Europhys. Lett. 66, 260 (2004).

${ }^{35}$ A. L. Vázquez de Parga, F. Calleja, B. Borca, M. C. G. Passeggi, J. J. Hinarejos, F. Guinea, and R. Miranda, Phys. Rev. Lett. 100, 056807 (2008)

${ }^{36}$ E. H. Hwang and S. Das Sarma, Phys. Rev. B 75, 205418 (2007).

${ }^{37}$ E. H. Hwang, R. Sensarma, and S. Das Sarma, Phys. Rev. B 82, 195406 (2010). 